\title{
Eficiencia reproductiva de alpacas machos en relación al tamaño testicular y niveles hormonales durante época reproductiva en puna seca
}

\section{Reproductive efficiency of male alpacas in relation to testes size and hormonal levels during the reproductive season in the dry puna}

\author{
Luis M. Incahuanaco ${ }^{1, a, *}$, Richard D. Ayala ${ }^{2, b}$, Rossi L. Hinojosa ${ }^{3, c}$, Edita Y. Torres ${ }^{4, d}$, \\ Teodosio Huanca ${ }^{1, e}$, Armando Nina ${ }^{1, \mathrm{f}}$, Julio Málaga ${ }^{1, \mathrm{~g}}$
}

\footnotetext{
${ }^{1}$ Programa Nacional de Camélidos, Instituto Nacional de Innovación Agraria, Estación Quimsachata- ILLPA, Puno, Perú.

2 Escuela de Posgrado, Programa de Maestría en Nutrición, Universidad Nacional Agraria la Molina, Lima, Perú.

${ }^{3}$ Facultad de ciencias agrarias, Universidad Nacional del Altiplano Puno, Puno, Perú.

${ }^{4}$ Laboratorio de Reproducción Animal, Facultad de Medicina Veterinaria y Zootecnia, Universidad Nacional del Altiplano Puno, Puno, Perú.

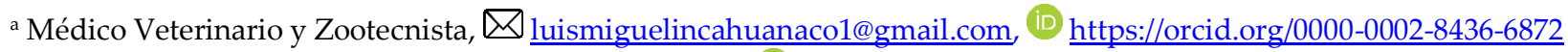

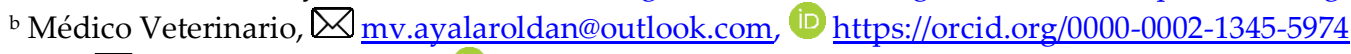

cIng., \luzrossih@gmail.com, iD https://orcid.org/0000-0002-2726-0254

${ }^{\mathrm{d}}$ Médico Veterinario y Zootecnista, $\$ torreseditayola7@gmail.com, (iD https://orcid.org/0000-0001-6956-3731

eDr., teodosio_huanca@yahoo.es, (iD https://orcid.org/0000-0001-5881-8671

${ }^{\mathrm{f}}$ Médico Veterinario y Zootecnista, $\bigotimes$ armandoo.mvz@gmail.com, iD https://orcid.org/0000-0001-5256-5781

g Dr., \imalaga@unap.edu.pe, iD https://orcid.org/0000-0003-4942-0041
}

* Autor de Correspondencia: Tel. +51 923017812

http://dx.doi.org/10.25127/riagrop.20212.678

http://revistas.untrm.edu.pe/index.php/RIAGROP revista.riagrop@untrm.edu.pe

Recepción: 29 de enero 2021

Aprobación: 26 de marzo 2021

Este trabajo tiene licencia de Creative Commons. Attribution-NonCommercial-ShareAlike $\quad 4.0$ International Public License - CC-BY-NC-SA 4.0

\section{Resumen}

El estudio se realizó en el CIP Quimsachata INIA - Puno, con objetivos de determinar la relación entre los niveles de testosterona y tamaño testicular de alpacas machos según edad y evaluar la fertilidad en alpacas hembras por efecto de frecuencia de monta. La sangre de doce alpacas machos fue analizada mediante RIA en el laboratorio de Reproducción Animal de la Universidad Cayetano Heredia - Lima. El tamaño testicular se midió con regla Vernier, las hembras se aparearon mediante empadre controlado y la fertilidad se evaluó con ecógrafo a los 41 días. La información fue analizada con Diseño Completo al Azar y Chi cuadrado. Los niveles de testosterona antes de la cópula en machos de 5 a 6 años fue $140.90 \pm 57.10 \mathrm{ng} / \mathrm{dL}$, 
los de 7 años $141.894 \pm 41.18 \mathrm{ng} / \mathrm{dL}$ y de 3 a 4 años $83.85 \pm 29.38 \mathrm{ng} / \mathrm{dL}$ ( $\mathrm{P} \leq 0.05)$. Después de la cópula, machos de 5 a 6 años $134.72 \pm 20.84$ ng/dL; 7 años $132.87 \pm 31.38$ ng/dL, y de 3 a 4 años $122.00 \pm 27.71$ ng/dL (P $\leq 0.05)$. Antes de la cópula, la testosterona en machos no vario según tamaño testicular ( $\mathrm{P} \geq 0.05)$. Después de la cópula, los machos con testículos menores a $2.5 \mathrm{~cm}$ produjeron $148.825 \pm 42.02 \mathrm{ng} / \mathrm{dL}$; con testículos de 2.5 a $3.5 \mathrm{~cm}$ $147.74 \pm 20.84 \mathrm{ng} / \mathrm{dL}$ y con testículos mayores a $3.5 \mathrm{~cm} 215.21 \pm 63.16 \mathrm{ng} / \mathrm{dL}(\mathrm{P} \leq 0.05)$. La fertilidad en hembras, con una cópula al día, fue de $40 \%$ con dos cópulas $55 \%$ las que se aparearon 3 veces $50 \%$ ( $\mathrm{P} \leq 0.05$ ).

Palabras claves: ecografia, empadre, fertilidad, testosterona,

\begin{abstract}
The study was carried out in CIP Quimsachata INIA - Puno, to determine the relationship between testosterone levels and testicular size of male alpacas according to age and evaluate the fertility in female alpacas by the effect of mating frequency; the blood of twelve male alpacas was analyzed by RIA in the Animal Reproduction laboratory of the Cayetano Heredia University - Lima. The testicular size was measured with the Vernier caliper, females were mated by a controlled mating system and fertility was evaluated with an ultrasound at 41 days. The information was analyzed with Complete Randomized Design and Chi-square. Testosterone levels before copulation in males aged 5 to 6 years were $140.90 \pm 57.10 \mathrm{ng} / \mathrm{dL}$, those of 7 years $141.894 \pm 41.18 \mathrm{ng} / \mathrm{dL}$ and those of 3 to 4 years $83.85 \pm 29.38 \mathrm{ng} / \mathrm{dL}(\mathrm{P} \leq 0.05)$; after copulation, males 5 to 6 years old $134.72 \pm 20.84 \mathrm{ng} / \mathrm{dL} ; 7$ years $132.87 \pm 31.38 \mathrm{ng} / \mathrm{dL}$, and from 3 to 4 years $122.00 \pm 27.71 \mathrm{ng} / \mathrm{dL}$ $(\mathrm{P} \leq 0.05)$. Before copulation, testosterone in males did not vary according to testicular size ( $\mathrm{P} \geq 0.05)$; after copulation, males with testes smaller than $2.5 \mathrm{~cm}$ produced $148.825 \pm 42.02 \mathrm{ng} / \mathrm{dL}$; with testes of 2.5 to $3.5 \mathrm{~cm}$ $147.74 \pm 20.84 \mathrm{ng} / \mathrm{dL}$ and with testes greater than $3.5 \mathrm{~cm} 215.21 \pm 63.16 \mathrm{ng} / \mathrm{dL}(\mathrm{P} \leq 0.05)$. Fertility in females with one copulation per day was $40 \%$ with two copulas $55 \%$ which mated 3 times $50 \%$ (P $\leq 0.05)$.
\end{abstract}

Keywords: ultrasound, mating system, fertility, testosterone.

\section{INTRODUCCIÓN}

El macho reproductor cumple una función importante en el proceso reproductivo en la mejora genética. Por lo tanto, depende de una adecuada selección, manejo, salud y alimentación, para el éxito de su crianza. Sin embargo, aún existen limitantes en el conocimiento científico relacionado a la evaluación de características seminales, tamaño testicular y hormonal (Huanca, 1998). Pues, no solamente la eficiencia reproductiva se basa sobre el comportamiento reproductivo de la hembra, además del índice de fertilidad, natalidad, preñez, entre otros; sino también, a estos indicadores que tienen una gran influencia la fertilidad del macho, existen factores adversos que limitan el desarrollo de la producción y productividad de la alpaca. Esto se refleja en los bajos índices de natalidad que oscila entre 55-60 \%. En este proceso, el macho juega un papel importante en el proceso reproductivo y mejoramiento genético, porque de su correcta selección y buen manejo dependerá el éxito de la explotación (Sumar, 1991). Por ello, el incremento del tamaño testicular se debe principalmente al aumento significativo del diámetro de los túbulos seminíferos y secundariamente por el incremento del volumen total del tejido intersticial, el desarrollo de los túbulos 
seminíferos en animales en proceso de alcanzar la pubertad es dependiente en gran parte del efecto de las hormonas gonadotróficas (LH y FSH), cuando el sistema nervioso central se hace menos sensible al efecto inhibidor de la testosterona (Hochereau de Reviers et al., 1993), La dimensión testicular constituye un importante indicador en la evaluación del potencial reproductivo del macho y puede ser empleado como criterio para predecir la producción diaria de semen debido a la elevada correlación encontrada entre la medición escrotal, el peso testicular y la producción total de semen (Skidmore, 2000).

Por lo tanto, se requiere seguir trabajando en el campo de la investigación para contribuir a mejorar los niveles reproductivos, si se tiene en cuenta que el $70 \%$ de los productores desarrollan una crianza tradicional y se sabe que las hembras de los camélidos sudamericanos tienen la limitante de producir sólo 4 o 5 crías durante toda su vida reproductiva. En la actualidad, la explotación de camélidos sudamericanos se lleva a cabo bajo sistemas tradicionales no siempre eficaces, que agudizan los problemas de morbilidad, mortalidad y baja eficiencia reproductiva; donde los porcentajes de natalidad anual en la mayoría de explotaciones alpaqueras es del orden del 50 \% (Fernández-Baca, 1993) con índices de fertilidad (Apaza et al., 2001) que no superan el $65 \%$, respectivamente. Para mejorar esta limitante, la aplicación de tecnologías como la ultrasonografía puede lograr una mayor eficiencia reproductiva en alpacas, para superar, de esta manera, la producción de crías logradas por alpaca hembra contribuir a lograr el incremento económico del productor alpaquero.

\section{MATERIALES Y MÉTODOS}

\subsection{Lugar de estudio}

El estudio se realizó en la estación experimental Quimsachata, perteneciente al Instituto Nacional de Innovación Agraria INIA-Puno, ubicado entre los distritos de Santa Lucia y Cabanillas de las provincias de Lampa y San Román del departamento de Puno. Este se encuentra a una altitud promedio de $4,200 \mathrm{~m}$, dentro de las coordenadas $15^{\circ} 04^{\prime}$ de latitud Sur, $70^{\circ} 18^{\prime}$ de longitud Oeste, donde la temperatura fluctúa entre $2{ }^{\circ} \mathrm{C}$ (mayo a julio) y $15{ }^{\circ} \mathrm{C}$ (septiembre a diciembre), localizado dentro de la zona agroecológica denominada puna seca (SENAMHI 2009).

\subsection{Animales en estudio}

La población estuvo conformada por alpacas machos de la raza huacaya, cuatro animales de tres a cuatro años, cuatro animales de cinco a seis años y cuatro animales mayor a siete años. $\mathrm{Al}$ respecto a las hembras, fueron utilizadas aquellas que presentaban celo el día del trabajo, que fueron un total de 114.

\subsection{Equipos, instrumentos y reactivos}

Se usó una regla vernier (0.5 mm. de precisión) y un ecógrafo marca Esaote Europe B.V. MOD 8100 REF 11280001 MREV.SN 16350099).

Se utilizó el kit de testosterona para radio inmuno análisis (RIA) de la marca comercial DIA source inmuno Asay S.A. USA.

\subsection{Obtención de muestras sanguíneas}

Se realizó la medida biométrica testicular, que incluyó el escroto. Los testículos fueron desplazados desde su ubicación normal hacia 
fuera, para mejorar su visualización de sus bordes y se fijaron con los dedos. Además, se procedió a medir el testículo tanto izquierdo y derecho, mediante la colocación de las puntas de la regla vernier en los dos extremos del testículo. El apareamiento que se manejó fue el de empadre controlado, el diagnóstico de gestación se realizó a los 41 días post empadre.

Se realizó la recolección de sangre antes y después del empadre, mediante venopunción yugular con una aguja de doble entrada, mediante tubos de vacutainer de $9 \mathrm{ml}$. que fueron rotulados previamente, la cantidad de sangre extraída es de $3 \mathrm{ml}$ aproximadamente. Las muestras después de colectarse fueron centrifugadas para la extracción del suero sanguíneo a $5000 \mathrm{rpm}$, por 5 minutos, posteriormente fueron almacenadas en congelación a $-20^{\circ} \mathrm{C}$, hasta su análisis.

\subsection{Análisis de los niveles de testosterona}

La determinación de los niveles de testosterona se realizó en las muestras de sangre recolectadas, mediante la técnica de radioinmuno análisis (RIA). La sensibilidad del ensayo fue de $0.01 \mathrm{ng} / \mathrm{dL}$ de testosterona en suero sanguíneo de acuerdo al protocolo recomendado por el fabricante, utilizando kits comerciales (DIA source inmuno Asay S.A. USA), la lectura de radiación se raelizó en un contador Gamma y los resultados se calcularon utilizando un sistema de reducción de datos para RIA.

\subsection{Análisis de datos}

Las informaciones obtenidas, sobre niveles de testosterona en alpacas machos, fueron analizadas mediante programa SAS (versión
9.3), adecuado al arreglo factorial de 2 (momento de cópula) x 3 (edades). Se utilizó correlación simple entre tamaño testicular y niveles de testosterona de las alpacas. Los datos sobre fertilidad en alpacas hembras por efecto de frecuencia de cópulas fueron analizados mediante la prueba estadística de Chi cuadrado.

\section{RESULTADOS Y DISCUSIÓN}

En la tabla 1, se muestra los resultados obtenidos del tamaño testicular en centímetros (cm) de alpacas reproductores de 3 a 4 años, 5 a 6 años y mayores a 7 años. Los tamaños testiculares por efecto de la edad de alpacas machos de 5 a 6 años mostraron $3.35 \pm 0.31 \mathrm{~cm}$ que fue similar a los de mayores a 7 años que tuvieron $3.07 \pm 0.688 \mathrm{~cm}$ y los que los de menor tamaño fueron de 3 a 4 años con $2.47 \pm 0.075 \mathrm{~cm}$.

Tabla 1. Tamaño testicular $(\mathrm{cm})$ de alpacas machos reproductores según edad

\begin{tabular}{cccc}
\hline Edad & N & Promedio \pm D.S. & $\begin{array}{c}\text { Valores } \\
\text { Extremos }\end{array}$ \\
\hline 3 a 4 años & 4 & $2.47 \pm 0.075^{\mathrm{b}}$ & $2.40-2.575$ \\
$\mathbf{5}$ a 6 años & 4 & $3.35 \pm 0.309^{\mathrm{a}}$ & $3.05-3.685$ \\
$\begin{array}{c}\text { Mayores a } \\
\text { 7 años }\end{array}$ & 4 & $3.07 \pm 0.688^{\mathrm{a}}$ & $2.43-3.72$ \\
\hline
\end{tabular}

Los valores encontrados en el presente estudio son inferiores a los reportados por Ramos (2014), quién registra medidas testiculares para los de 3 años $3.12 \mathrm{~cm}$; 4 años $3.17 \mathrm{~cm}$; años $3.11 \mathrm{~cm}$; 6 años $3.22 \mathrm{~cm}$; 7 años $3.14 \mathrm{~cm}$ y 8 años $3.32 \mathrm{~cm}(\mathrm{P} \geq 0.05)$. En época lluviosa, es $3.29 \mathrm{~cm}$ y en época seca, $3.07 \mathrm{~cm}$., según zonas agroecológicas, en Puna húmeda fue $3.10 \mathrm{~cm}$ y para alpacas de puna seca fue $3.02 \mathrm{~cm}$. También Bravo (2000) reporta las dimensiones 
testiculares en la alpaca adulta, con un promedio de $3.05 \mathrm{~cm}$. Por otro lado, García et al. (2005) reportaron un promedio de 2.75 a 3.75 $\mathrm{cm}$, que comparado al tamaño testicular encontrado en el presente estudio $(3.35 \mathrm{~cm}$.) resultan similares.

\subsection{Niveles de testosterona}

Los niveles de testosterona en los machos reproductores, por efecto de edad y antes de la cópula, en los machos de 5 a 6 años mostraron $140.90 \pm 57.10 \mathrm{ng} / \mathrm{dL}$ de testosterona en suero sanguíneo, que fue similar con alpacas mayores a 7 años que mostraron $141.89 \pm 41.18 \mathrm{ng} / \mathrm{dL}$ y los de menor tamaño fueron de 3 a 4 años con $83.86 \pm 29.38 \mathrm{ng} / \mathrm{dL}$. Posterior a la cópula, los machos de 5 a 6 años mostraron $134.72 \pm 20.84$ $\mathrm{ng} / \mathrm{dL}$, que fue similar con los de mayores a 7 años que mostraron $132.87 \pm 31.38 \mathrm{ng}$ y los de menor tamaño fueron de 3 a 4 años con $122.00 \pm$ $27.71 \mathrm{ng} / \mathrm{dL}$ (tabla 2).

Los valores encontrados en este estudio son inferiores al reporte de Carpio (1999), donde los animales de 2 años mostraron niveles de testosterona de $216.30 \mathrm{ng} / \mathrm{dL}$ en suero sanguíneo, los de 3 años produjeron 538.50 ng/dL y los 3.5 años 524.70 ng/dL.

Cebra et al. (2015) supera a los resultados del presente estudio, en animales de 2 años, que mostraron niveles de testosterona $216.30 \mathrm{ng} / \mathrm{dL}$ en suero sanguíneo, los de 2.5 años produjeron $283.50 \mathrm{ng} / \mathrm{dL}$, los de 3 años mostraron 538.30 ng/dL y los mayores a 3.5 años mostraron 524.70 ng/dL en suero sanguíneo. También se aprecia que, a medida que aumenta la edad de los reproductores, incrementa el tamaño testicular y por ende el aumento de los niveles de testosterona en llamas y alpacas tal como menciona Cebra et al., (2015).

\subsection{Fertilidad de alpacas}

La fertilidad en alpacas hembras por efecto del número de cópulas del macho; en donde los machos que aparearon una vez por día mostraron fertilidad de $40 \%$. Además, los que aparearon dos veces por día alcanzaron $55.0 \%$ y las que aparearon 3 veces logró 50\%, obteniendo 12, 33 y 12 alpacas preñadas respectivamente (tabla 3 ).

Los índices de fertilidad encontrados en el presente estudio son inferiores a los reportes de Apaza et al. (1999), quien reporta fertilidades de 85 a $90 \%$ con una sola cópula en forma diaria mediante empadre controlado, trabajos realizados en la comunidad de Huanacamaya y CIP Quimsachata de la zona puna seca. En CIP La Quimsachata, la tasa de concepción promedio al primer servicio fue del $57.86 \%$ (Calsin, 2011). La tasa de concepción al primer servicio en alpacas en condiciones de puna seca desde 1998 al 2003 fue de $68.81 \%$, con tendencia disminuir en relación al tiempo (Pari, 2005). En el CIP Chuquibambilla, al evaluar los índices reproductivos en alpacas primerizas de la raza Suri, la tasa de concepción al primer servicio fue de $42.73 \%$ (Olarte et al. 2009). La tasa de fertilidad está relacionada con el número de servicios por día, que es mayor cuando se realizan menor número de servicios por día. Por esto, se deduce que la menor tasa de concepción se debe también a una disminución de la tasa de la efectividad de los servicios en el mismo día (Bravo et al. 1995). 
Tabla 2. Niveles de testosterona ( $\mathrm{ng} / \mathrm{dL}$ ) de alpacas reproductores según edad y momentos de cópula (antes y después)

\begin{tabular}{ccccc}
\hline Momentos de cópula & Edad /años & N & Promedio (ng/dL) & D. S. \\
\hline \multirow{2}{*}{ Antes } & 3 a 4 & 4 & $83.86^{\mathbf{b}}$ & 29.38 \\
& 5 a 6 & 4 & $140.90^{\mathrm{a}}$ & 57.10 \\
& Mayores a 7 & 4 & $141.89^{\mathrm{a}}$ & 41.18 \\
\hline \multirow{2}{*}{ Después } & 3 a 4 & 4 & $122.00^{\mathrm{b}}$ & 27.71 \\
& 5 a 6 & 4 & $134.72^{\mathrm{a}}$ & 20.84 \\
& Mayores a 7 & 4 & $132.87^{\mathrm{a}}$ & 31.38 \\
\hline
\end{tabular}

Tabla 3. Fertilidad de alpacas hembras según frecuencia de cópulas

\begin{tabular}{ccccc}
\hline Reproductores machos & $\begin{array}{c}\text { Número de } \\
\text { reproductores }\end{array}$ & $\begin{array}{c}\mathbf{N}^{\circ} \text { total } \\
\text { cópulas }\end{array}$ & $\mathbf{N}^{\circ}$ preñadas & \% preñadas \\
\hline Primera copula & 30 & 30 & 12 & 40.0 \\
Segunda copula & 30 & 60 & 33 & 55.0 \\
Tercera copula & 8 & 24 & 12 & 50.0 \\
\hline
\end{tabular}

\section{CONCLUSIONES}

El tamaño testicular varía por edad del macho. La concentración de testosterona en los machos incrementó tanto antes y después de la cópula y se evidenció que a mayor tamaño testicular, mayor cantidad de testosterona por efecto de la alimentación y clima.

La fertilidad en alpacas hembras por efecto de frecuencia de cópulas fue de 40, 55 y $50 \%$ con 1 , 2 y 3 cópulas por día, respectivamente y a mayor frecuencia de copula, igual porcentaje de preñez.

\section{Referencias}

Apaza, N., R. Sapana y T. Huanca, (2001). Inseminación artificial en alpacas con semen fresco en comunidades campesinas. Rev Invest Vet, Perú Supl. 1: 435-438.
Bravo W, A. Skidmore, y X. Zhao, (2000). Reproductive aspects and storage of semen in Camelidae. Anim Reprod Sci 62: 173-193. doi : 10.1016/ S03784320(00)00158-5

García, W., D. Pezo, F. San Martín, J. Olazábal y F. Franco, (2005). Manual del Técnico Alpaquero. Instituto Veterinario de Investigaciones Tropicales y de Altura (NITA). Estación Experimental La Raya. Universidad Nacional Mayor de San Marcos. Lima.

Calsin, B. (2011) eficiencia reproductiva y repercusión económica en la crianza de alpacas huacaya del centro de investigación y producción Quimsachata INIA puno. Tesis de maestría. Facultad medicina veterinaria y zootecnia. Universidad Nacional del Altiplano Puno. 74 pp.

Carpio, M. (1999). Presencia de espermatozoides, Niveles de testosterona y tamaño testicular en alpacas, II congreso mundial Sobre camélidos sudamericanos Cusco Perú.

Cebra C., A. Tibary y J.saun, (2015) llama and alpaca care. Edit. Elseiver, 1ra edición, Canada.

Fernández-Baca, S. (1993). Incremento reproductivo en alpacas de explotaciones comerciales mediante método de empadre alternado. Rev. Inv. Pec. IVIT A., Universidad mayor de San Marcos, Lima. 
Hochereau de Reviers, MT., AC. Locatelli, C. Pisselet y BP. Setchell, (1993) Effects of a single brief period of moderate heating of the testes on seminiferous tubules in hypophysectomized rams treated with pituitary extract. J Reprod Fert; 97: 381- 387.

Huanca, W. (1998). Importancia de la Evaluación Reproductiva de Alpaca macho resumen XXI APPA 98. Universidad Nacional del Altiplano, Puno- Perú. Pp. 167-169.

Olarte, C., R. Rojas, N. Luque y L. Condori, (2009). Eficiencia reproductiva en alpacas de la raza Suri. En: Resúmenes y Trabajos del V Congreso mundial sobre camélidos Riobamba-Ecuador.

Pari, R. (2005). Principales factores individuales sobre algunos parámetros reproductivos en alpacas hembras de puna seca en el anexo experimental
Quimsachata. Tesis para optar el Título de Médico Veterinario y Zootecnista Tesis. Facultad de Medicina Veterninaria y Zootecnia Universidad Nacional del Altiplano Puno.

Ramos, J. (2014). Tamaño testicular según edad, época y zona agroecológica en alpacas (Vicugna paco)". Tesis para optar el título profesional de Médico Veterinario y Zootecnista - Universidad Nacional de Altiplano - Puno.

Sumar, J. (1991). Avances y perspectivas del conocimiento de los camélidos sudamericanos. Revista FAO. Pp. 114-117 Santiago-Chile.

Skidmore, L. (2000). Anatomía del tracto reproductivo en camélidos. Recent advances in camelid reproduction. segunda edicion, editorial ivis, Ithaca, New York. USA. 